\title{
Robust Stability of System with M Input and N Output in a Class of Catastrophe "Hyperbolic Umbilic"
}

\author{
Beisenbi Mamyrbek, Mukataev Nurlan \\ Department of System Analysis and Control, L.N.Gumilyov Eurasian National University, \\ 010000 Kazakhstan, Astana, 2, Mirzoyana str. \\ beisenbi_ma@enu.kz,sgpi@mail.ru
}

\begin{abstract}
The robust stability of control systems where the controlled object has dynamics is highlighted in present. This paper proposes a new approach to designing control systems for objects with uncertain parameters in the form of three-parameter structurally stable mappings. This method allows the synthesis of highly effective control system, which has wide field of robust stability. For a dynamic system affected by parametric uncertainty, this paper focuses on robust stability analysis of system with Lyapunov functions. This paper presents some theoretical fundamental results assisting in analyzing of the behavior of control systems, depending on parameter uncertainty. The obtained results are robust stability type since the robust stability is guaranteed under certain deviations from the current state. We developed a new method for the study of robust stability MIMO linear automatic control systems with $\boldsymbol{m}$ inputs and $\boldsymbol{n}$ - output by constructing a vector Lyapunov function. Using the approach to the construction of Lyapunov functions, we have asymptotically stable stationary state of the control object. In fact, the results to provide dynamic safety and performance of control systems. Finally, conditions for robust stability is presented permitting to understand the application of the proposed approach.

Index Terms - control system, uncertainty of parameters, robust stability, structurally stable mappings, stability area, method of Lyapunov functions, catastrophe.
\end{abstract}

\section{Introduction}

The modern problems of control is characterized by escalating complexity of control objects, the requirement of high efficiency and stability in the numerous uncertainty conditions and incomplete information.

At present almost in all spheres of production and equipment control systems are widely applied: in mechanical engineering, energetics, the electronic, chemical, metallurgical, biological and textile industry, transport, a robotics, aircraft, space systems, high-precision military technology and equipment. In these systems uncertainty can be caused as existence of the uncontrollable indignations operating on control object, and ignorance of true values of parameters of control objects and their unpredictable change in time. In this regard, creation of the control systems providing somewhat the best protection against uncertainty in knowledge of object properties is one of the actual problems. Ability of a control system to keep stability in the conditions of parametrical or nonparametric uncertainty is understood as a robust system [1-4].

For the solution of problems of robust stability we offer a universal approach to the creation of Lyapunov's vector functions [5-7]. Components of a an anti-gradient vector of vector functions from geometrical interpretation of the theorem of the Lyapunov's second method [8-10] are set by components of a speed vector (the right member of equation of a state). Researches of robust stability of the system are made by designing of a negative function [11-14] which is equal to a scalar product of a gradients vector on a speed vector. Stability conditions turn out from positive definiteness of a Lyapunov's vector function, in the form of inequalities system in uncertain parameters of control objects and established parameters of the regulator. The method of research the robust stability of MIMO linear systems of automatic control with $\mathrm{m}$ - input and with $\mathrm{n}$ - outputs on the basis of construction a Lyapunov's vector function is developed and conditions of robust stability are received.

In scientific literature [1-4] there are some well-known methods of creation of objects control systems with uncertain parameters, which are generally devoted to the determination of robust stability of system with the set structure of linear laws of control or inertialess nonlinear (relay) characteristics and do not allow to project a control system with rather wide area of robust stability in the conditions of big uncertainty of parameters of control object and drift of their characteristic in big limits.

Nowadays scientific provisions on development and research of control systems with rather wide area of robust stability do not exist.

The problem described in the article is the problem of creation of a robust steady control system by dynamic objects, with uncertain parameters with approach to synthesis of control systems in a class of the structural and stable mappings [15-17], allowing to maximize the potential of robust stability and indicators of quality of a control system.

\section{Mathematical Formulation of Model}

Let us investigate the problem of creation of control systems with an increased potential of robust stability [18-21] in a class three-parameter structurally stable mappings (catastrophe hyperbolic umbilic) [22-24] for linear objects with $\mathrm{m}$ input and $\mathrm{n}$ outputs.

The control system is described by the following equation:

$$
\begin{aligned}
& x(t)=A x(t)+B u(t) \\
& y(t)=C x(t)
\end{aligned}
$$

Variables: $x(t) \in R^{n}$ vector of a system's condition, 
$u(t) \in R^{m}$ control vector, $y(t) \in R^{e}$ system exit. $A \in R^{n \times n}$, $B \in R^{n \times m}, C \in R^{1 \times n}$ matrixes do not depend on $t$ time. Let us consider a case of nonlinear control of linear object and we will assume that it is a vector - function $u(t)=u(\cdot) \in R^{m}$

Therefore we will assume that $y=x$ the condition of system is known. The purpose of control is the choice of such forms of nonlinear vector functions from a class of threeparameter structurally stable mappings (catastrophe hyperbolic umbilic) [23,24] which give to system (1) set properties (stability at any change of uncertain parameters) systems.

The law of control is set in the form of three-parameter structurally stable mappings (catastrophe hyperbolic umbilic) [15-16],[20-21],[23-24].

$$
u_{i}=-x_{i}^{3}-x_{i+1}^{3}-k_{i, i+1} x_{i} x_{i+1}+k_{i} x_{i}+k_{i+1} x_{i+1} i=1, \ldots, n ;
$$

A linear object and B control Matrixes respectively are shown below:

$$
A=\left\|\begin{array}{cccc}
a_{11} & a_{12} & , \ldots, & a_{1 n} \\
a_{21} & a_{22} & , \ldots, & a_{2 n} \\
\ldots & \ldots & \ldots & \ldots \\
a_{n 1} & a_{n 2} & , \ldots, & a_{n n}
\end{array}\right\|, \quad B=\left\|\begin{array}{cccc}
b_{11} & 0 & \ldots, & 0 \\
0 & b_{22} & , \ldots, & 0 \\
\ldots & \ldots & \ldots & \ldots \\
0 & 0 & \ldots, & b_{n n}
\end{array}\right\|
$$

\section{Stationary Conditions of System}

The installed states of systems (1) will be defined by the solution of the equations:

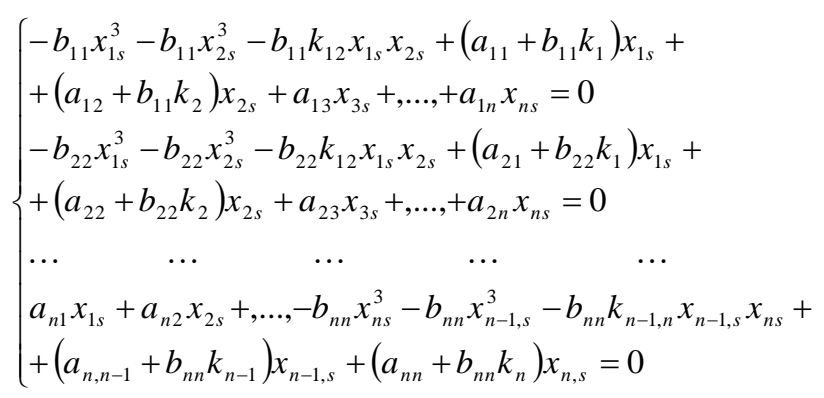

From system of the equations (3) we can find stationary states

$$
x_{1 s}=0, x_{2 s}=0, \ldots, x_{n s}=0 \text {, }
$$

Some other stationary states can be defined by the solution of the equations:

$$
-b_{i i} x_{i s}^{2}+a_{i i}+b_{i i} k_{i}=0, \quad x_{j s}=0 \text { if } i \neq j, i=1, \ldots, n
$$

The equation (5) at negative, $k_{i}+\frac{a_{i i}}{b_{i i}} \quad i=1, \ldots, n$, $\left(k_{i}+\frac{a_{i i}}{b_{i i}}<0, i=1, \ldots, n\right)$ has imaginary decisions that can't correspond to any physical possible situation. When $\left(k_{i}-\frac{a_{i i}}{b_{i i}}\right)$ more than zero $\left(k_{i}-\frac{a_{i i}}{b_{i i}}>0\right)$ the equation (5) allows the following established states:

$$
\begin{aligned}
& x_{i s}=\sqrt{k_{i}+\frac{a_{i i}}{b_{i i}}}, x_{j s}=0 \text { if } i \neq j, i=1, \ldots, n ; j=1, \ldots, n \\
& \quad \text { and } \\
& x_{i s}=-\sqrt{k_{i}+\frac{a_{i i}}{b_{i i}}}, x_{j s}=0 \text { if } i \neq j, i=1, \ldots, n ; j=1, \ldots, n
\end{aligned}
$$

\section{Research of Stability}

Let us research Stability of stationary conditions (4), (6) and (7) of systems (1) by the functions of Lyapunov's method.

1. Let us consider stability of a steady state (4).

Let us enter designation of components of anti-gradient vector from a function vector $V(x)=\left(V_{1}(x), V_{2}(x), \ldots, V_{n}(x)\right)$ :

$$
\begin{aligned}
& \frac{\partial V_{1}(x)}{\partial x_{1}}=b_{11} x_{1}^{3}+\frac{1}{2} b_{11} k_{12} x_{1} x_{2}-b_{11}\left(k_{1}+\frac{a_{11}}{b_{11}}\right) x_{1} \\
& \frac{\partial V_{1}(x)}{\partial x_{2}}=b_{11} x_{1}^{3}+\frac{1}{2} b_{11} k_{12} x_{1} x_{2}-b_{11}\left(k_{1}+\frac{a_{11}}{b_{11}}\right) x_{1} \\
& \frac{\partial V_{1}(x)}{\partial x_{3}}=-a_{13} x_{3}, \frac{\partial V_{1}(x)}{\partial x_{4}}=-a_{14} x_{4}, \ldots, \frac{\partial V_{1}(x)}{\partial x_{n}}=-a_{1 n} x_{n} \\
& \frac{\partial V_{2}(x)}{\partial x_{1}}=b_{22} x_{1}^{3}+\frac{1}{2} b_{22} k_{12} x_{1} x_{2}-b_{22}\left(k_{1}+\frac{a_{21}}{b_{22}}\right) x_{1} \\
& \frac{\partial V_{2}(x)}{\partial x_{2}}=b_{22} x_{2}^{3}+\frac{1}{2} b_{22} k_{22} x_{1} x_{2}-b_{22}\left(k_{2}+\frac{a_{22}}{b_{22}}\right) x_{2} \\
& \frac{\partial V_{2}(x)}{\partial x_{3}}=-a_{23} x_{3}, \frac{\partial V_{2}(x)}{\partial x_{4}}=-a_{24} x_{4}, \ldots, \frac{\partial V_{2}(x)}{\partial x_{n}}=-a_{2 n} x_{n}
\end{aligned}
$$$$
\begin{aligned}
& \cdots \quad \cdots \quad \cdots \quad \cdots \\
& \frac{\partial V_{n}(x)}{\partial x_{1}}=-a_{n 1} x_{1}, \frac{\partial V_{n}(x)}{\partial x_{2}}=-a_{n 2} x_{2} \\
& \frac{\partial V_{n}(x)}{\partial x_{3}}=-a_{n 3} x_{3}, \ldots, \frac{\partial V_{n}(x)}{\partial x_{n-1}}=b_{n n} x_{n}^{3}+\frac{1}{2} b_{n n} k_{n-1, n} x_{n-1} x_{n}-b_{n n}\left(k_{n}+\frac{a_{n n}}{b_{n n}}\right) x_{n} \\
& \frac{\partial V_{n}(x)}{\partial x_{n}}=b_{n n} x_{n}^{3}+\frac{1}{2} b_{n n} k_{n-1, n} x_{n-1} x_{n}-b_{n n}\left(k_{n}+\frac{a_{n n}}{b_{n n}}\right) x_{n}
\end{aligned}
$$

Full derivative of time from Lyapunov's function $V(x)=\left(V_{1}(x), V_{2}(x), \ldots, V_{n}(x)\right)$ we will receive in as follows: 


$$
\begin{aligned}
& \frac{d V(x)}{d t}=-b_{11}^{2}\left[\begin{array}{l}
x_{1}^{3}+\frac{1}{2} k_{12} x_{1} x_{2}- \\
\left(k_{1}+\frac{a_{11}}{b_{11}}\right) x_{1}
\end{array}\right]^{2}-b_{11}^{2}\left[\begin{array}{l}
\left.x_{2}^{3}+\frac{1}{2} k_{12} x_{1} x_{2}-\right]^{2}- \\
\left(k_{2}+\frac{a_{12}}{b_{11}}\right) x_{2}
\end{array}\right]^{-} \\
& -a_{13}^{2} x_{3}^{2}-, \ldots,-a_{1 n}^{2} x_{n}^{2}-b_{22}^{2}\left[x_{1}^{3}+\frac{1}{2} k_{12} x_{1} x_{2}-\left(k_{2}+\frac{a_{21}}{b_{22}}\right) x_{1}\right]^{2}- \\
& -b_{22}^{2}\left[x_{2}^{3}+\frac{1}{2} k_{12} x_{1} x_{2}-\left(k_{2}+\frac{a_{22}}{b_{22}}\right) x_{2}\right]^{2}-a_{23}^{2} x_{3}^{2}-, \ldots,-a_{2 n}^{2} x_{n}^{2}-, \ldots,-a_{n 1}^{2} x_{1}^{2}- \\
& -a_{n 2}^{2} x_{2}^{2}-, \ldots,-b_{n n}^{2}\left[x_{n-1}^{3}+\frac{1}{2} k_{n-1, n} x_{n-1} x_{n}-\left(k_{n-1}-\frac{a_{n, n-1}}{b_{n n}}\right) x_{n-1}\right]^{2}- \\
& -b_{n n}^{2}\left[x_{n}^{3}+\frac{1}{2} k_{n-1, n} x_{n-1} x_{n}-\left(k_{n}-\frac{a_{n n}}{b_{n n}}\right) x_{n}\right]^{2}
\end{aligned}
$$

From (8) it is visible that the full derivative of time from Lyapunov's vector function will be negative function, therefore, the sufficient condition of asymptotic stability of system is satisfied.

On components of an anti-gradient Lyapunov's function in a scalar form is represented as:

$$
\begin{aligned}
& V(x)=V_{1}(x)+V_{2}(x)+, \ldots,+V_{n}=\frac{1}{4} b_{11} x_{1}^{4}+\frac{1}{4} b_{11} k_{12} x_{1}^{2} x_{2}-\frac{1}{2} b_{11}\left(k_{1}+\frac{a_{11}}{b_{22}}\right) x_{1}^{2}+\frac{1}{4} b_{11} x_{2}^{4}+ \\
& +\frac{1}{4} b_{11} k_{12} x_{1} x_{2}^{2}-\frac{1}{2} b_{11}\left(k_{2}+\frac{a_{12}}{b_{11}}\right) x_{2}-\frac{1}{2} a_{13} x_{3}^{2}-, \ldots,-\frac{1}{2} a_{1 n} x_{n}^{2}+\frac{1}{4} b_{22} x_{1}^{4}+\frac{1}{4} b_{22} k_{12} x_{1}^{2} x_{2}- \\
& -\frac{1}{2} b_{22}\left(k_{1}+\frac{a_{21}}{b_{22}}\right) x_{1}^{2}+\frac{1}{4} b_{22} x_{2}^{4}+\frac{1}{4} b_{22} k_{12} x_{1} x_{2}^{2}-\frac{1}{2} b_{22}\left(k_{2}+\frac{a_{22}}{b_{22}}\right) x_{2}^{2}-\frac{1}{2} a_{23} x_{3}^{2}-, \ldots,- \\
& -\frac{1}{2} a_{2 n} x_{n}^{2}+, \ldots,-\frac{1}{2} a_{n 1} x_{1}^{2}-\frac{1}{2} a_{n 2} x_{2}^{2}-, \ldots,-\frac{1}{2} a_{n, n-2} x_{n-2}^{2}+\frac{1}{4} b_{n n} x_{n-1}^{4}+\frac{1}{4} b_{n n} k_{n-1, n} x_{n-1}^{2} x_{n}- \\
& -\frac{1}{2} b_{n n}\left(k_{n-1}+\frac{a_{n, n-1}}{b_{n n}}\right) x_{n-1}^{2}+\frac{1}{4} b_{n n} x_{n}^{4}+\frac{1}{4} b_{n n} k_{n-1, n} x_{n-1} x_{n}^{2}-\frac{1}{2} b_{n n}\left(k_{n}+\frac{a_{n n}}{b_{n n}}\right) x_{n}^{2}=\frac{1}{4}\left(b_{11}+b_{22}\right) x_{1}^{4}+ \\
& +\frac{1}{4} k_{12}\left(b_{11}+b_{22}\right) x_{1}^{2} x_{2}+\frac{1}{4}\left(b_{11}+b_{22}\right) x_{2}^{4}+\frac{1}{4} k_{12}\left(b_{11}+b_{22}\right) x_{1} x_{2}^{2}-\frac{1}{2}\left[\left(b_{11}+b_{22}\right) k_{1}+a_{11}+a_{21}\right] x_{1}^{2}- \\
& -\frac{1}{2}\left[\left(b_{11}+b_{22}\right) k_{2}+a_{12}+a_{22}\right] x_{2}^{2}-\frac{1}{2}\left[\left(b_{11}+b_{22}\right) k_{1}+a_{11}+a_{21}+a_{31}+, \ldots, a_{n 1}\right] x_{1}^{2}- \\
& -\frac{1}{2}\left[\left(b_{11}+b_{22}\right) k_{2}+a_{12}+a_{22}+a_{32}+, \ldots, a_{n 2}\right] x_{2}^{2}+\frac{1}{4}\left(b_{33}+b_{44}\right) x_{3}^{4}+\frac{1}{4} k_{34}\left(b_{33}+b_{44}\right) x_{3}^{2} x_{4}+ \\
& +\frac{1}{4}\left(b_{33}+b_{44}\right) x_{4}^{4}+\frac{1}{4} k_{34}\left(b_{33}+b_{44}\right) x_{3} x_{4}^{2}-\frac{1}{2}\left[\left(b_{33}+b_{44}\right) k_{3}+a_{13}+a_{23}+, \ldots, a_{n 3}\right] x_{3}^{2}- \\
& -\frac{1}{2}\left[\left(b_{33}+b_{44}\right) k_{4}+a_{14}+a_{24}+, \ldots, a_{n 4}\right] x_{4}^{2}+, \ldots,+\frac{1}{4}\left(b_{n-1, n-1}+b_{n n}\right) x_{n-1}^{4}+ \\
& +\frac{1}{4} k_{n-1, n}\left(b_{n-1, n-1}+b_{n n}\right) x_{n-1}^{2} x_{4}+\frac{1}{4}\left(b_{n-1, n-1}+b_{n n}\right) x_{n}^{4}+\frac{1}{4} k_{n-1, n}\left(b_{n-1, n-1}+b_{n n}\right) x_{n-14}^{4} x_{n}^{2}- \\
& -\frac{1}{2}\left[\left(b_{n-1, n-1}+b_{n n}\right) k_{n-1}+a_{1, n-1}+a_{2, n-1}+a_{3, n-1}+, \ldots, a_{n, n-1}\right] x_{n-1}^{2}- \\
& \frac{1}{4}\left[\left(b_{n-1, n-1}+b_{n n}\right) k_{n}+a_{1 n}+a_{2 n}+a_{3 n}+, \ldots,+a_{n n}\right] x_{n}^{2}
\end{aligned}
$$

Conditions of positive or negative definiteness of Lyapunov's functions of $V(x)$ from (9) are not obvious, therefore we can use the Mors lemma from theories of accidents $[23,24]$.

From the Mors lemma (9) we can locally present Lyapunov's function (5) to vicinities of a steady state in the form of a square form taking into account the equation of a state (3):

$$
\begin{aligned}
& V_{2 j}(x)=-\left[\left(b_{11}+b_{22}\right) k_{1}+a_{11}+a_{21}+a_{31}+, \ldots, a_{n 1}\right] x_{1}^{2}- \\
& -\left[\left(b_{11}+b_{22}\right) k_{2}+a_{12}+a_{22}+a_{32}+, \ldots, a_{n 2}\right] x_{2}^{2}- \\
& -\left[\left(b_{33}+b_{44}\right) k_{3}+a_{13}+a_{23}+a_{33}+, \ldots, a_{n 3}\right] x_{3}^{2}- \\
& -\left[\left(b_{33}+b_{44}\right) k_{4}+a_{14}+a_{24}+a_{34}+, \ldots, a_{n 4}\right] x_{4}^{2}-, \ldots,- \\
& -\left[\left(b_{n-1, n-1}+b_{n n}\right) k_{n-1}+a_{1, n-1}+a_{2, n-1}+a_{3, n-1}+, \ldots, a_{n, n-1}\right] x_{n-1}^{2}- \\
& -\left[\left(b_{n-1, n-1}+b_{n n}\right) k_{n}+a_{1 n}+a_{2 n}+a_{3 n}+, \ldots, a_{n n}\right] x_{n}^{2}
\end{aligned}
$$

Conditions of positive definiteness of a square form (10) (stability of a steady state (5)) are defined by system of inequalities:

$$
\left\{\begin{array}{l}
a_{11}+a_{21}+a_{31}+, \ldots,+a_{n 1}+b_{11} k_{1} b_{22} k_{1}<0 \\
a_{12}+a_{22}+a_{32}+, \ldots,+a_{n 2}+b_{11} k_{2} b_{22} k_{2}<0 \\
a_{13}+a_{23}+a_{33}+, \ldots,+a_{n 3}+b_{33} k_{3} b_{44} k_{3}<0 \\
a_{14}+a_{24}+a_{34}+, \ldots,+a_{n 4}+b_{33} k_{4} b_{44} k_{4}<0 \\
\ldots \quad \ldots \quad \ldots \quad \ldots \quad \ldots \\
a_{1, n-1}+a_{2, n-1}+a_{3, n-1}+, \ldots,+a_{n, n-1}+b_{n-1, n-1} k_{n-1}+b_{n n} k_{n-1}<0 \\
a_{1 n}+a_{2 n}+a_{3 n}+\ldots,+a_{n n}+b_{n-1, n-1} k_{n}+b_{n n} k_{n}<0
\end{array}\right.
$$

Thus, the area of stability of a steady state (4) will be defined by performance of system of the inequalities (11), made rather uncertain parameters of object control and chosen parameters of a control system.

2. Let us investigate stability of stationary states (6) and (7). The equations of a state (1) will be represented in deviations of rather steady state (6) and (7), and by known formalism [22].

Equations of a state (1) in deviations of steady state (6) an (7) write them down, as:

$$
\begin{aligned}
& \int \dot{x}_{1}=-b_{11} x_{1}^{3}-b_{11} x_{2}^{3}-b_{11} k_{12} x_{1} x_{2}-3 b_{11} \sqrt{k_{1}+\frac{a_{11}}{b_{11}} x_{1}^{2}}-3 b_{11} \sqrt{k_{2}+\frac{a_{12}}{b_{11}} x_{2}^{2}}- \\
& -2 b_{11}\left(k_{1}+\frac{a_{11}}{b_{11}}\right) x_{1}-2 b_{11}\left(k_{2}+\frac{a_{12}}{b_{11}}\right) x_{2}+a_{13} x_{3}+, \ldots,+a_{1 n} x_{n} \\
& \dot{x}_{2}=-b_{22} x_{1}^{3}-b_{22} x_{2}^{3}-b_{22} k_{12} x_{1} x_{2}-3 b_{22} \sqrt{k_{1}+\frac{a_{21}}{b_{11}} x_{1}^{2}}-3 b_{22} \sqrt{k_{2}+\frac{a_{22}}{b_{22}} x_{2}^{2}}- \\
& -2 b_{22}\left(k_{1}+\frac{a_{21}}{b_{22}}\right) x_{1}-2 b_{22}\left(k_{2}+\frac{a_{22}}{b_{22}}\right) x_{2}+a_{23} x_{3}+, \ldots,+a_{2 n} x_{n} \\
& \left\{\dot{x}_{3}=-a_{31} x_{1}-a_{32} x_{2}+a_{33} x_{3}^{3}-b_{33} x_{4}^{3}-b_{33} k_{34} x_{3} x_{4}-3 b_{33} \sqrt{k_{3}+\frac{a_{21}}{b_{11}} x_{1}^{2}}-\right. \\
& -3 b_{33} \sqrt{k_{4}+\frac{a_{34}}{b_{33}} x_{4}^{2}}-2 b_{33}\left(k_{3}+\frac{a_{33}}{b_{33}}\right) x_{3}-2 b_{33}\left(k_{4}+\frac{a_{34}}{b_{33}}\right) x_{4}+, \ldots,+a_{3 n} x_{n} \\
& \begin{array}{lcccc}
\cdots & \cdots & \cdots & \cdots & \cdots \\
\dot{x}_{n}=-a_{n 1} x_{1}-a_{n 2} x_{2}+, \ldots, b_{n n} x_{n-1}^{3}-b_{n n} x_{n}^{3}-b_{n n} k_{n-1, n} x_{n-1} x_{n}-
\end{array} \\
& -3 b_{n n} \sqrt{k_{n-1}+\frac{a_{n, n-1}}{b_{n-1, n}} x_{n-1}^{2}}-3 b_{n n} \sqrt{k_{n}+\frac{a_{n n}}{b_{n n}} x_{n}^{2}}-2 b_{n n}\left(k_{n-1}+\frac{a_{n, n-1}}{b_{n n}}\right) x_{n-1}- \\
& -2 b_{n n}\left(k_{n}+\frac{a_{n n}}{b_{n n}}\right) x_{n}
\end{aligned}
$$

Let us accept components of an anti-gradient vector from vector of functions $V(x)=\left(V_{1}(x), V_{2}(x), \ldots, V_{n}(x)\right)$, by the equal: 


$$
\begin{aligned}
& \frac{\partial V_{1}(x)}{\partial x_{1}}=b_{11} x_{1}^{3}+\frac{1}{2} b_{11} k_{12} x_{1} x_{2}+3 b_{11} \sqrt{k_{1}+\frac{a_{11}}{b_{11}} x_{1}^{2}}+2 b_{11}\left(k_{1}+\frac{a_{11}}{b_{11}}\right) x_{1} ; \\
& \frac{\partial V_{1}(x)}{\partial x_{2}}=b_{11} x_{2}^{3}+\frac{1}{2} b_{11} k_{12} x_{1} x_{2}+3 b_{11} \sqrt{k_{2}+\frac{a_{12}}{b_{11}} x_{2}^{2}}+2 b_{11}\left(k_{2}+\frac{a_{12}}{b_{11}}\right) x_{2} ; \\
& \frac{\partial V_{1}(x)}{\partial x_{3}}=-a_{13} x_{3}, \ldots, \frac{\partial V_{1}(x)}{\partial x_{3}}=-a_{1 n} x_{n} \text {; } \\
& \frac{\partial V_{2}(x)}{\partial x_{1}}=b_{22} x_{1}^{3}+\frac{1}{2} b_{22} k_{12} x_{1} x_{2}+3 b_{22} \sqrt{k_{1}+\frac{a_{21}}{b_{22}} x_{1}^{2}}+2 b_{22}\left(k_{1}+\frac{a_{21}}{b_{22}}\right) x_{1} ; \\
& \frac{\partial V_{2}(x)}{\partial x_{1}}=b_{22} x_{2}^{3}+\frac{1}{2} b_{22} k_{12} x_{1} x_{2}+3 b_{22} \sqrt{k_{2}+\frac{a_{22}}{b_{22}} x_{2}^{2}}+2 b_{22}\left(k_{2}+\frac{a_{22}}{b_{22}}\right) x_{2} \text {; } \\
& \frac{\partial V_{2}(x)}{\partial x_{3}}=-a_{23} x_{3}, \ldots, \frac{\partial V_{2}(x)}{\partial x_{n}}=-a_{2 n} x_{n} \text {; } \\
& \frac{\partial V_{n}(x)}{\partial x_{1}}=-a_{n 1} x_{1}, \frac{\partial V_{n}(x)}{\partial x_{2}}=-a_{2 n} x_{n}, \ldots, \\
& \frac{\partial V_{n}(x)}{\partial x_{n}}=b_{n n} x_{n}^{3}+\frac{1}{2} b_{n n} k_{n-1, n} x_{n-1} x_{n}+3 b_{n n} \sqrt{k_{n}+\frac{a_{n n}}{b_{n n}} x_{n}^{2}}+2 b_{n n}\left(k_{n}+\frac{a_{n n}}{b_{n n}}\right) x_{n} ; \\
& \int \frac{d V_{1}(x)}{d t}=\frac{\partial V_{1}(x)}{\partial x} \frac{d x_{1}}{d t}=-b_{11}^{2}\left[x_{1}^{3}+\frac{1}{2} k_{12} x_{1} x_{2}+3 \sqrt{k_{1}+\frac{a_{11}}{b_{11}}} x_{1}^{2}+2\left(k_{1}+\frac{a_{11}}{b_{11}}\right) x_{1}\right]^{2}- \\
& -b_{11}^{2}\left[x_{2}^{3}+\frac{1}{2} k_{12} x_{1} x_{2}+3 \sqrt{k_{2}+\frac{a_{12}}{b_{11}}} x_{2}^{2}+2\left(k_{2}+\frac{a_{12}}{b_{11}}\right) x_{2}\right]^{2}-a_{13}^{2} x_{3}^{2}, \ldots,-a_{1 n}^{2} x_{n}^{2} \\
& \frac{d V_{2}(x)}{d t}=\frac{\partial V_{2}(x)}{\partial x} \frac{d x_{2}}{d t}=-b_{22}^{2}\left[x_{1}^{3}+\frac{1}{2} k_{12} x_{1} x_{2}+3 \sqrt{k_{1}+\frac{a_{21}}{b_{22}}} x_{1}^{2}+2\left(k_{1}+\frac{a_{21}}{b_{22}}\right) x_{1}\right]^{2}- \\
& -b_{22}^{2}\left[x_{2}^{3}+\frac{1}{2} k_{12} x_{1} x_{2}+3 \sqrt{k_{2}+\frac{a_{22}}{b_{22}}} x_{2}^{2}\right]-a_{23}^{2} x_{3}^{2}-, \ldots,-a_{2 n}^{2} x_{n}^{2}
\end{aligned}
$$

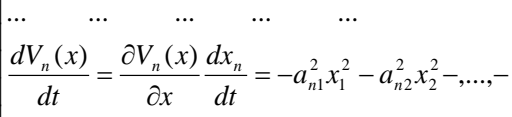

$$
\begin{aligned}
& -b_{n n}^{2}\left[x_{n-1}^{3}+\frac{1}{2} k_{n-1, n} x_{n-1} x_{n}+3 \sqrt{k_{n-1}-\frac{a_{n, n-1}}{b_{n n}}} x_{n-1}^{2}+2\left(k_{n-1}-\frac{a_{n, n-1}}{b_{n n}}\right) x_{n-1}\right]^{2}- \\
& -b_{n n}^{2}\left[x_{n}^{3}+\frac{1}{2} k_{n-1, n} x_{n-1} x_{n}+3 \sqrt{k_{n}-\frac{a_{n n}}{b_{n n}}} x_{n}^{2}+2\left(k_{n}-\frac{a_{n n}}{b_{n n}}\right) x_{n}\right]^{2}
\end{aligned}
$$

From (13) it is visible that the full derivative on time from Lyapunov's vector function will be negative function, therefore, the sufficient condition of asymptotic stability of system (12) is satisfied.
Lyapunov's function in a scalar form it is representable in a view:

$$
\begin{aligned}
& V(x)=V_{1}(x)+V_{2}(x)+, \ldots,+V_{n}(x)=\frac{1}{4} b_{11} x_{1}^{4}+\frac{1}{4} b_{11} k_{12} x_{1}^{2} x_{2}+ \\
& +b_{11} \sqrt{k_{1}+\frac{a_{11}}{b_{11}} x_{1}^{3}}+b_{11}\left(k_{1}+\frac{a_{11}}{b_{11}}\right) x_{1}^{2}+\frac{1}{4} b_{11} x_{2}^{4}+\frac{1}{4} b_{11} k_{12} x_{1} x_{2}^{2}+ \\
& +b_{11} \sqrt{k_{2}+\frac{a_{12}}{b_{11}} x_{2}^{3}}+b_{11}\left(k_{2}+\frac{a_{12}}{b_{11}}\right) x_{2}^{2}-\frac{1}{2} a_{12} x_{3}^{2}-, \ldots,-a_{1 n} x_{n}^{2}+ \\
& +\frac{1}{4} b_{22} x_{1}^{4}+\frac{1}{4} b_{22} k_{12} x_{1}^{2} x_{2}+b_{22} \sqrt{k_{1}+\frac{a_{21}}{b_{22}} x_{1}^{3}}+b_{22}\left(k_{1}+\frac{a_{21}}{b_{22}}\right) x_{1}^{2}+ \\
& +\frac{1}{4} b_{22} x_{2}^{4}+\frac{1}{4} b_{22} k_{12} x_{1} x_{2}^{2}+b_{22} \sqrt{k_{2}+\frac{a_{22}}{b_{22}} x_{2}^{3}}+b_{22}\left(k_{2}+\frac{a_{22}}{b_{22}}\right) x_{2}^{2}- \\
& -\frac{1}{2} a_{23} x_{3}^{2}-, \ldots,-\frac{1}{2} a_{2 n} x_{n}^{2}+, \ldots,-\frac{1}{2} a_{n-1} x_{1}^{2}-\frac{1}{2} a_{n 2} x_{n}-, \ldots,+ \\
& +\frac{1}{4} b_{n n} x_{n-1}^{4}+\frac{1}{4} b_{n n} k_{n-1} x_{n-1}^{2} x_{n}+b_{n n} \sqrt{k_{n-1}+\frac{a_{n-1, n}}{b_{n n}} x_{n-1}^{3}} \\
& +b_{n n}\left(k_{n-1}+\frac{a_{n-1, n}}{b_{n n}}\right) x_{n-1}^{2}+\frac{1}{4} b_{n n} x_{n}^{4}+\frac{1}{4} b_{n n} k_{n-1, n} x_{n-1} x_{n}^{2}+ \\
& +b_{n n} \sqrt{k_{n}+\frac{a_{n n}}{b_{n n}} x_{n}^{3}}+b_{n n}\left(k_{n}+\frac{a_{n n}}{b_{n n}}\right) x_{n}^{2}
\end{aligned}
$$

Conditions of positive or negative definiteness of functions (14) are unevident therefore we will use the Morse's lemma from the theory of catastrophe.

On a lemma of the Mors of Lyapunov's function (15) in a vicinity of a steady state (6) and (7) it is locally possible to present in the form of a square form taking into account the equation of a state (12) in deviations of rather steady state (6) or (7):

$$
\begin{aligned}
& V(x) \approx\left[\left(b_{11}+b_{22}\right) k_{1}+a_{11}+a_{21}-\frac{1}{2}\left(a_{31}+a_{41}+, \ldots,+a_{n}\right)\right] x_{1}^{2}+ \\
& +\left[\left(b_{11}+b_{22}\right) k_{2}+a_{12}+a_{22}-\frac{1}{2}\left(a_{32}+a_{42}+, \ldots,+a_{n 2}\right)\right] x_{2}^{2}+ \\
& +\left[\left(b_{33}+b_{44}\right) k_{3}+a_{33}+a_{43}-\frac{1}{2}\left(a_{13}+a_{23}+, \ldots, a_{n 3}\right)\right] x_{3}^{2}+ \\
& +\left[\left(b_{33}+b_{44}\right) k_{4}+a_{44}+a_{34}-\frac{1}{2}\left(a_{14}+a_{24}+, \ldots,+a_{n 4}\right)\right] x_{4}^{2}+, \ldots,+ \\
& +\left[\left(b_{n-1, n-1}+b_{n n}\right) k_{n-1}+a_{n-1, n-1}+a_{n, n-1}-\frac{1}{2}\left(a_{1, n-1}+a_{2, n-1}+a_{3, n-1}+, \ldots,+a_{n, n-1}\right)\right] x_{n-1}^{2}+ \\
& +\left[\left(b_{n-1, n-1}+b_{n n}\right) k_{n}+a_{n-1, n}+a_{n n}-\frac{1}{2}\left(a_{1 n}+a_{2 n}+a_{3 n}+, \ldots,+a_{n-2, n}\right)\right] x_{n}^{2}
\end{aligned}
$$

Stability conditions of a steady state (6) or (7) are defined by positive definiteness of a square form (15) i.e. system of inequalities: 


$$
\left\{\begin{array}{l}
\left(b_{11}+b_{22}\right) k_{1}+a_{11}+a_{21}>\frac{1}{2}\left(a_{31}+a_{41}+, \ldots,+a_{n 1}\right) \\
\left(b_{11}+b_{22}\right) k_{2}+a_{12}+a_{22}>\frac{1}{2}\left(a_{32}+a_{42}+, \ldots,+a_{n 2}\right) \\
\left(b_{33}+b_{44}\right) k_{3}+a_{33}+a_{43}>\frac{1}{2}\left(a_{13}+a_{23}+, \ldots,+a_{n 3}\right) \\
\left(b_{33}+b_{44}\right) k_{4}+a_{34}+a_{44}>\frac{1}{2}\left(a_{14}+a_{24}+, \ldots,+a_{n 4}\right) \\
\ldots \quad \ldots \quad \ldots \quad \ldots \quad \ldots \quad \ldots \quad \ldots \quad+a_{n, n-1}>\frac{1}{2}\left(a_{1, n-1}+a_{2, n-1}+, \ldots,+a_{n, n-1}\right) \\
\left.\left(b_{n-1, n-1}+b_{n n}\right) k_{n-1}+a_{m-1, m-1}+a_{n-1, n}\right) \\
\left(b_{n-1, n-1}+b_{n n}\right) k_{n}+a_{n-1, n}+a_{n n}>\frac{1}{2}\left(a_{1 n}+a_{2 n}+, \ldots,+a_{n-2, n}\right)
\end{array}\right.
$$

Thus, MIMO control system, constructed in a class of three-parameter structurally stable mappings, will be steady in beyond all bounds wide limits of change of uncertain parameters of object control.

The steady state (4) exists and is steady at change of uncertain parameters in area (11), and stationary states (6) and (7) exist at loss of stability of a steady state (4) and they at the same time don't exist.

Steady stationary states (6) and (7) appear when performing inequalities (16).

\section{Conclusions}

As a universal method of research of stability of dynamic systems is considered the method of A.M.Lyapunov's functions. As the instrument of research in Lyapunov's method some special continuously differentiable are used, turning at the beginning of coordinates in zero the functions called Lyapunov's function. Application of this method restrains lack of universal approach to creation of Lyapunov's function. It is necessary to remind that the mistake in a choice and failure to construct necessary Lyapunov's function doesn't mean instability of system: it points only to failure at creation of Lyapunov's function.

Use of the developed approach to create Lyapunov's functions allows to demonstrate that the system has asymptotically steady stationary states both in negative, and in positive area of change of uncertain parameters of object control. Upon transition of uncertain parameters through zero there is a bifurcation and new steady branches appear. This is zero steady state loses stability.

These stationary states at the same time do not exist and there is an opportunity to synthesize steady system at any change of uncertain parameters.

Actually the results received on creation of control systems with an increased potential of robust stability, allow to ensure dynamic safety and operability of operated systems at a stage of their construction and exploitation.

\section{References}

[1] Polyak B.T., Scherbakov P. S. Robust stability and management. - M.: Science, 2002. -303 pages.
[2] Safonof M.G. Stability and robustness of multivariable feedback systems. - Cambridge, MA: MIT Press, 1980.

[3] Chen M.J., Desoer C.A. Necessary and sufficient for robust stability of linear distributed feedback systems // Intern. J. Control. - 1982. - V.35, No.2. - P.255-267.

[4] Besekersky V.A. Nebylov A.V. Robust system of automatic control. - M.: Science, 1983. - 239 pages.

[5] Beisenbi M.A., Kulniyazova K.S., Research of robust stability in control system with Lyapunov direct method, Proceedings of 11-th InterUniversity Conference on Mathematics and Mechanics. - Astana, Kazakhstan. 2007.- pp. 50-56.

[6] Beisenbi M., Yermekbayeva J. The Research of the Robust Stability in Linear System. // International Conference on Control, Engineering \& Information Technology (CEIT'13), Sousse, Tunisia, 2013. - Proceedings of IPCO. - P.142-147.

[7] Beisenbi M.A., Yermekbayeva J.J., Beisenbin A.M. The New Control Method of the Research Robust Stability for Linear System. Life Science Journal 2013;10 (12s): - P. 142-148.

[8] Barbashin E.A. Introduction in the stability theory - M.: Science, 1967. 225 pages.

[9] Malkin I.G. Theory of stability of movement. - M.: Science, 1966. - 540 pages.

[10] Voronov A.A., Matrosov V. M. Metod of Lyapunov's vector functions in the stability theory - M.: Science, 1987. -312 pages.

[11]Beisenbi Mamyrbek, Uskenbayeva Gulzhan. The New Approach of Design Robust Stability for Linear Control System. Bangkok, Thailand. Publisher: Institute of Research engineers and Doctors. Conference on Advances in Electronics and Electrical Technology. AEET 2014. Copyright (C) Institute of Research Engineers and Doctors. ISBN: 978981-07-8859-9 doi: 10.3850/ 978-981-07-8859-9 57. p. 11-18.

[12]Beisenbi M.A., Uskenbayeva G.A. Method of Lyapunov's function in research of robust stability of linear control system with one input and one output .// A miscellany of material XV MNPK "Prospects for the development of information technology".- Novosibirsk, 2013. - p.27-32

[13]Beisenbi M.A., Satibaldina D.K., Uskenbayeva G.A., Research of robust stability of linear SISO system by method of A.M.Lyapunov function // Herald ENU, Astana, № 6 (97) 2013., -p. 117-125.

[14]Beisenbi M.A., Satibaldina D.K., Uskenbayeva G.A., Research of robust stability of control system of linear objects by $m x n$ method of A.M.Lyapunov function // Herald ENU, Astana, № 6 (97) 2013., - p 36-45.

[15]Beisenbi M. A. Methods of potential increase of robust stability of control systems. - Astana, 2011. - 352 pages.

[16]Beisenbi M. A. Models and methods of the system analysis and management of the determined chaos in economy. - Astana, 2011. - 201 pages.

[17]Beisenbi M. A., Erzhanov B. A. Control systems with an increased potential of robust stability. - Astana, 2002. - 164 pages.

[18]Beisenbi M.A., Abdrakhmanova L.G. Research of dynamic properties of control systems with increased potential of robust stability in a class of two-parameter structurally stable maps by Lyapunov function. // International Conference on Computer, Network and Communication Engineering (ICCNCE 2013). - Published by Atlantis Press, 2013. - P. 201-203.

[19]Beisenbi M.A., Abrahmanova L.G. Research of control systems with increased potential of robust stability by Lyapunov function.// A miscellany of material XV MNPK "Prospects for the development of information technology". - Novosibirsk, 2013. - p. 22-37.

[20]Beisenbi M., Mukataev N., Robust stability of system with one input and one output in a class of catastrofhe "hyperbolic umbilic". // International Conference on Control, Engineering \& Information Technology (CEIT'14), Sousse, Tunisia, 2014. - Proceedings of IPCO.

[21]Beisenbi M.A., Mukataev N.S. Robust stability of system with one input and one output in a class of catastrophe "hyperbolic umbilic".// Science journal "Young scientist"- Kazan, 2014.-№2(61). - p.107-117.

[22] Gregoire Nicolis, Ilya Prigogine. Exploring Complexity an Introduction. - New York, 1989.

[23]Poston G., Stewart I. Theory of accidents and its appendix. - M.: World, 1980.

[24] Gilmore R. Applied theory of accidents. T.1. - M.: World, 1981. 